\title{
Tendances actuelles des réformes éducatives
}

Juan Carlos Tedesco

\section{OpenEdition}

Journals

Édition électronique

URL : http://journals.openedition.org/ries/4307

DOI : 10.4000/ries.4307

ISSN : 2261-4265

\section{Éditeur}

Centre international d'études pédagogiques

\section{Édition imprimée}

Date de publication : 1 mars 1994

Pagination : 77-85

ISSN : 1254-4590

\section{Référence électronique}

Juan Carlos Tedesco, "Tendances actuelles des réformes éducatives », Revue internationale

d'éducation de Sèvres [En ligne], 01 | 1994, mis en ligne le 20 avril 2015, consulté le 01 mai 2019. URL http://journals.openedition.org/ries/4307; DOI : 10.4000/ries.4307

Ce document a été généré automatiquement le 1 mai 2019.

(c) Tous droits réservés 


\title{
Tendances actuelles des réformes éducatives
}

\author{
Juan Carlos Tedesco
}

1 Ce texte ${ }^{1}$ vise à résumer les principales leçons tirées du processus de changement éducatif mis en place au cours des dernières décennies. Il présente une série de réflexions fondées sur l'information et l'expérience rassemblées sur le plan de la coopération internationale. À cet égard, il est nécessaire de préciser que ces réflexions sont principalement inspirées des expériences des pays d'Amérique latine et d'Europe et des États-Unis. Le cas des pays asiatiques mérite une considération particulière, en raison de l'influence significative de leurs facteurs culturels en général et du rôle de la famille en particulier. Dans le cas des pays d'Afrique, en plus de l'importance également significative des facteurs culturels, l'analyse du changement éducatif est intimement associée au problème plus général des conditions de vie matérielles de la population qui ont une incidence directe sur les chances de succès de toute stratégie d'apprentissage quelle qu'elle soit. Les pays arabes présentent des situations proches de certaines de celles qui précèdent, sauf dans le cas des pays où s'exerce une forte influence de l'intégrisme islamique et où les changements éducatifs sont associés au problème de la lutte entre traditionalisme et modernisation.

\section{Les effets pervers des changements permanents de l'éducation}

2 Au cours des trois dernières décennies au moins, et pour faire face aux nouveaux défis du développement social, le système éducatif a, dans de nombreux pays, fait l'objet de propositions de réformes successives et changeantes qui ont, paradoxalement, renforcé sa rigidité et son immobilisme.

3 Pour cette raison, on observe maintenant dans certains secteurs politiques et sociaux un profond scepticisme quant aux possibilités de modifier le fonctionnement du système éducatif. Un coup d'œil rapide à l'histoire des dernières décennies permet de soutenir que 
tout a été essayé dans le domaine de l'éducation et que les résultats sont néanmoins très minces.

4 Ce scepticisme est particulièrement sensible en ce qui concerne les possibilités de changement de l'intérieur du système et provoqué par ses propres acteurs. Les enseignants et le reste du personnel de l'éducation ont tendance à être perçus chaque fois plus fortement comme un élément du problème et non de la solution. Les explications de ce phénomène sont variées et bien connues: changements permanents décidés sans consultation ni évaluation des résultats, déprofessionnalisation du personnel - accentuée pendant la dernière décennie surtout dans les pays en développement par la diminution des salaires résultant des politiques d'ajustement - et renforcement des comportements corporatifs.

5 En conséquence, la nécessité de surmonter le scepticisme concernant les possibilités de modifier le fonctionnement des administrations éducatives est un des premiers obstacles à tout processus de réforme éducative. Les stratégies de changement radical provenant de l'extérieur du système provoquent la résistance des acteurs à l'intérieur. Des stratégies fondées exclusivement sur la capacité interne d'auto transformation sont très lentes et finissent généralement par céder aux pressions pour satisfaire les demandes corporatives. En conséquence, il existe actuellement une tendance à placer la priorité du changement éducatif sur l'aspect institutionnel. L'objectif consiste à ouvrir les institutions aux besoins de la société et à introduire des facteurs de dynamisme dans les mécanismes internes de la gestion éducative. En résumé, il s'agit de favoriser une gestion qui présente une plus grande capacité de réaction face aux demandes et aux résultats.

\section{Les conditions du succès : consensus national et long terme}

6 Une des leçons apprises du « réformisme permanent » déjà évoqué est que les politiques éducatives ne sont pas des politiques à court terme, ni des politiques qui relèvent exclusivement d'un seul secteur. Le succès des stratégies éducatives dépend, entre autres facteurs, de la continuité de leur application. Mais pour que la continuité soit assurée, il est nécessaire d'obtenir, dans l'application de ces stratégies, le consensus et l'engagement de tous les acteurs.

7 Le débat international démontre que des conditions favorables existent actuellement pour établir de nouvelles alliances autour des stratégies d'éducation. Des secteurs qui, par le passé, avaient des intérêts différents tendent aujourd'hui à converger vers certains objectifs de l'éducation de base qui peuvent ainsi se transformer en objectifs de consensus national. Responsabiliser la société dans son ensemble envers les actions éducatives implique de lui accorder l'autorité nécessaire pour définir des orientations. Dans ce sens, les politiques d'éducation doivent dépasser le stade des politiques gouvernementales et assumer le titre qui leur a été donné de politiques d'État. La traduction pratique de cette tendance est, paradoxalement, la revalorisation de certaines formules gouvernementales en matière d'éducation propres aux origines de nos systèmes: conseils nationaux d'éducation comportant une pluralité de représentation soumise au consensus national, conseils d'éducation locaux, autonomie des établissements, etc. En résumé, la gestion de l'éducation doit être à l'abri de toute influence de parti. 
8 Reconnaître que les politiques de l'éducation sont des politiques à long terme implique également d'admettre que pour définir leurs stratégies il faut avoir une grande capacité d'anticipation par rapport aux demandes et aux problèmes futurs. Du point de vue politique, la capacité d'anticipation exige un accord qui oriente le comportement des acteurs en fonction des objectifs nationaux. Cependant, si l'accord politique est une condition nécessaire, il n'est pas suffisant en soi. La capacité d'anticipation exige également de disposer de diagnostics confirmés sur la situation existante, d'un large éventail d'informations sur les tendances mondiales et de mécanismes d'évaluation des résultats des actions entreprises qui permettent le cas échéant d'adopter une autre trajectoire avant que certains résultats ne se consolident et que leur correction soit difficile et coûteuse. Pour cette raison, le renforcement des systèmes d'information (mesure des résultats, analyse des tendances internationales, etc.) constitue actuellement un domaine d'activité des plus intenses dans le remaniement des systèmes de gestion éducative.

\section{Les ressources financières: condition indispensable mais non suffisante du changement}

9 Le manque de ressources financières a été un des arguments les plus souvent cités pour expliquer les résultats limités de l'action éducative. Il ne fait aucun doute que, dans la majorité des pays, les ressources consacrées à l'éducation sont rares et, de plus, sujettes à des variations constantes en raison de l'instabilité politique ou de l'inflation. Cependant, la comparaison internationale indique que même dans des cas où les ressources financières existaient, les résultats de l'éducation ont également été insatisfaisants. L'exemple des États-Unis est, probablement, le plus éloquent. À cet égard, la citation suivante est sans commentaire :

Pendant plus de trois décennies, depuis l'inauguration par les Soviétiques du premier satellite au monde, le Spoutnik, les gouvernements successifs des ÉtatsUnis se sont efforcés (parfois de façon très énergique) d'améliorer la situation. Les dépenses réelles par élève ont atteint un taux de croissance annuel de 3,75\%, triplant quasiment entre 1960 et 1988. De plus, les coûts additionnels ont provoqué exactement ce que demandaient les défenseurs de dépenses plus élevées: des classes réduites et des maîtres mieux formés et expérimentés. Pendant le dernier quart de siècle, le rapport moyen élève/maître dans les écoles publiques est passé de plus de 25 à moins de 18 , soit une réduction de $30 \%$. Plus de la moitié des enseignants actuels ont au moins un Master. En 1986 déjà, la moitié des enseignants avaient un minimum de quinze années d'expérience de l'enseignement. Ces données, considérées dans leur ensemble, illustrent l'importance réelle du problème. Les coûts ont triplé et le rendement a baissé. Les ressources additionnelles ont clairement augmenté les dépenses du système éducatif par rapport au passé, cependant cela ne paraît pas l'avoir amélioré. Les États-Unis ne peuvent espérer inverser les tendances de l'éducation en déboursant simplement plus d'argent. À notre avis, ils devraient entreprendre une réforme qui modifie en profondeur la façon de dépenser. Cette nation doit étudier attentivement l'organisation de l'éducation ${ }^{2}$.

La question du financement de l'éducation mérite une analyse séparée. Elle est mentionnée ici uniquement comme un élément additionnel qui confirme l'hypothèse de la nécessité d'un changement global de l'éducation et de l'inutilité de continuer à doter le système existant de ressources supplémentaires. 


\section{Les réformes éducatives n'ont plus pour objectif l'augmentation quantitative mais le changement et l'amélioration qualitative}

11 Le concept selon lequel des années d'étude plus nombreuses sont associées de façon linéaire à une plus grande productivité et à une plus grande participation sociale est dépassé. Les efforts réalisés pour augmenter l'impact quantitatif du système éducatif ont été considérables. Toutefois, les pays où l'expansion quantitative a été réussie constatent aujourd'hui que les résultats obtenus ne sont pas proportionnés aux efforts consentis tant par les gouvernements que par les familles : des taux de redoublement très élevés ou des résultats de l'apprentissage très bas indiquent que même pour résoudre les problèmes quantitatifs il est nécessaire de modifier la qualité de l'offre pédagogique.

12 L'accent mis sur la qualité est actuellement imposé par les changements observés dans les demandes des sociétés envers l'éducation. Les modifications technologiques et structurelles sur les lieux de travail, ainsi que le renforcement de la démocratie politique, exigent un comportement du citoyen fondé sur le développement de certaines capacités que les systèmes éducatifs traditionnels ne véhiculent pas de façon systématique: maitrise des codes de la circulation de l'information, capacité de traiter cette dernière, de résoudre les problèmes, de travailler en équipe, d'exprimer des besoins.

13 Le problème consiste à définir comment s'enseignent ou se développent ces capacités. L'expérience apportée par les réformes du curriculum et les réformes pédagogiques appliquées à ce jour pose au moins deux grandes interrogations.

14 La première a trait à la possibilité de définir des politiques relatives aux méthodes d'apprentissage. L'expérience internationale démontre qu'il existe une grande diversité de procédés et que les méthodes sont définies par les enseignants, même dans les cas où un règlement et des normes exhaustives sont en vigueur. La solution de rechange à une stratégie de définition de politiques ou de normes pour les méthodes d'apprentissage consiste à renforcer le professionnalisme des enseignants par la formation initiale et continue.

15 La seconde interrogation est techniquement plus complexe. À ce jour, les théories de l'apprentissage ont relativement bien résolu la question du processus d'acquisition des concepts scientifiques ou de l'information. Mais les nouveaux défis posent celle de savoir comment s'apprennent ou se développent les capacités, les valeurs, les attitudes. À cet égard, les réponses sont moins catégoriques, tant du point de vue de la théorie de l'apprentissage que de ses applications dans les institutions scolaires.

16 L'accent sur la qualité s'exprime actuellement par le souci des résultats de l'apprentissage. Un des aspects centraux des tendances actuelles de la réforme de l'éducation consiste, précisément, à établir des mécanismes efficaces pour l'évaluation des résultats.

\section{La demande éducative comme facteur de changement}

17 La focalisation des réformes éducatives sur le changement de l'offre est une de leurs caractéristiques les plus importantes. Le rôle de la demande a été sous-estimé ou n'a été 
pris en compte que dans certaines étapes initiales du processus de changement. Un des traits les plus novateurs des tendances actuelles de la réforme éducative est, au contraire, le rôle actif accordé à la demande sociale. Cette tendance s'exprime à travers deux types d'actions différents :

1. mesures et programmes destinés à donner un plus grand pouvoir de décision aux usagers du système éducatif (parents ou élèves) grâce à des mécanismes spécifiques (vouchers, autonomie institutionnelle, privatisation);

2. programmes destinés à améliorer la qualité de la demande en éducation, grâce à une meilleure information des utilisateurs.

\section{La dimension systémique du changement}

18 Les stratégies de réforme adoptées par le passé étaient caractérisées par leur focalisation sur la modification d'un facteur déterminé, considéré comme facteur clé du changement de l'éducation: augmentation des salaires des enseignants, réformes des contenus, remaniement administratif (décentralisation), équipement, infrastructures, etc. L'évaluation de ces changements a permis d'observer que leurs résultats modestes s'expliquent parce qu'ils ont eu lieu de façon relativement isolée du reste des autres facteurs. Les changements en éducation dépendent de l'interaction de facteurs multiples qui agissent de façon systémique.

19 Reconnaitre ce caractère systémique ne signifie cependant pas qu'il est nécessaire ou possible de modifier tout en même temps. Cela signifie en revanche qu'à un certain moment il est nécessaire de prendre conscience des conséquences de la modification d'un facteur spécifique sur les autres facteurs. Une mesure de changement institutionnel - la décentralisation, par exemple - qui ne prévoit pas à quel moment et selon quelles orientations auront lieu la formation du personnel, la réforme des contenus, la révision des échelles salariales ou des méthodes pour la fourniture de l'équipement et du matériel didactique, aura certainement un impact réduit sur les résultats éducatifs.

Le problème central des réformes consiste donc à établir la séquence et l'étendue des changements de chacun des facteurs. L'expérience démontre que ces aspects (séquence et étendue) peuvent être plus facilement définis sur le plan local qu'au niveau central. Il est pratiquement impossible de déterminer une séquence de réformes éducatives similaires dans des contextes sociaux, géographiques et culturels très différents. Pour cette raison, il existe actuellement une forte tendance à accorder la priorité aux changements institutionnels visant à offrir une plus grande autonomie aux institutions et à leur permettre de définir leurs propres stratégies d'amélioration (projet d'établissement, autonomie institutionnelle, etc.).

\section{Le changement institutionnel est une priorité}

Le point clé du débat sur la transformation institutionnelle est, évidemment, le rôle de l'État. À cet égard, l'analyse comparative internationale permet également de constater qu'il n'y a pas de solution simple ni facile telle que l'étatisation ou la privatisation, la centralisation ou la décentralisation. L'expérience internationale démontre que dans les cas où la décentralisation est réussie, elle a eu lieu à partir d'une administration centrale 
bien établie, de même que les cas de privatisation réussie sont associés à la présence d'administrations étatiques solides.

En définitive, la question est de déterminer clairement le rôle de l'administration centrale-étatique. À cet égard, le résumé des débats actuels permet de définir au moins trois grands domaines de responsabilité :

- le premier a trait à la détermination des priorités par des mécanismes de discussion démocratique ;

- le deuxième se réfère à l'établissement et à la mise en œuvre de mécanismes qui permettent d'évaluer les résultats obtenus et l'application de ces priorités, accordant un grand niveau d'autonomie aux institutions et instances locales pour définir les processus grâce auxquels ces résultats seront obtenus ;

- le troisième, particulièrement conséquent dans des pays caractérisés par des déséquilibres sociaux importants, est lié à l'application de mécanismes efficaces de compensation des différences qui neutralisent les risques antidémocratiques des stratégies de décentralisation.

En résumé, du point de vue stratégique, le changement du type d'organisation institutionnelle par l'intermédiaire de laquelle sont offerts les services éducatifs est une priorité. Une plus grande autonomie des institutions éducatives et un plus grand contrôle des résultats, accompagnés de mécanismes de compensation qui garantissent l'équité, paraissent être le fondement des transformations les plus prometteuses dans ce domaine.

\section{Réforme ou innovation institutionnelle?}

La tendance vers une autonomie institutionnelle plus large implique la modification du schéma traditionnel de "réforme du système " pour se concentrer sur les innovations institutionnelles ou interinstitutionnelles. Dans les systèmes éducatifs de tradition centralisatrice, l'innovation se limitait au secteur privé ou à des "expériences pilotes " dans le secteur public. Dans le meilleur des cas, les expériences réussies servaient de base à des mesures de changement générales, entraînant des résultats qui n'étaient pas toujours très favorables. Actuellement, il est de plus en plus admis que le succès des innovations dépend de leur adéquation aux conditions locales de leur application. Pour cette raison, il paraît plus important de généraliser la capacité d'innover plutôt que les innovations elles-mêmes. Les innovations fructueuses jouent un rôle important du point de vue de la généralisation de la capacité d'innover, si elles sont utilisées comme exemple dans la formation du personnel. De ce point de vue, il est également évident que la capacité d'innover est étroitement associée au professionnalisme du personnel.

\section{La question de la privatisation}

$\mathrm{Au}$ cours des dernières années, un débat animé a eu lieu concernant la privatisation en tant que stratégie du changement en éducation. Le débat se situait, évidemment, dans le contexte plus général de la privatisation des entreprises et des services publics. Toutefois, la spécificité de l'action éducative et de ses effets sociaux fait que cette question mérite une considération très attentive. L'information disponible permet, au moins, de tirer les conclusions suivantes :

En premier lieu, il n'y a pas une relation univoque entre la privatisation de l'éducation, le développement économique et la modernisation. Des pays ayant un pourcentage élevé 
d'enseignement privé atteignent des résultats aussi satisfaisants que des pays où existe une forte prédominance étatique et, à l'inverse, une plus grande étendue de l'enseignement privé n'entraîne pas des résultats plus satisfaisants, sur le plan économique ni éducatif. En fait, globalement, le secteur de l'enseignement privé est plus important dans les pays en développement que dans les pays développés.

Ensuite, les bons résultats de l'éducation sont généralement associés à des facteurs de type institutionnel: existence d'un projet pédagogique, direction, travail en équipe, responsabilité des résultats. Tous ces facteurs et possibilités sont normalement associés au fonctionnement d'institutions éducatives privées. Cependant, là où le secteur public offre ces possibilités à ses institutions, les résultats sont également satisfaisants. En conséquence, le problème est de parvenir à introduire le dynamisme du secteur privé dans la gestion du système éducatif public, sans que ce dernier perde son caractère démocratique. Ce défi nous ramène, à nouveau, à la question de l'autonomie institutionnelle, au contrôle des résultats et à la compensation des différences.

\section{La capacité d'innover et l'autonomie institutionnelle sont-elles le propre des pays riches?}

La réforme éducative fondée sur une autonomie et une responsabilité plus larges des acteurs locaux implique d'admettre la nécessité d'un niveau plus élevé de personnalisation des services. À cet égard, il faut reconnaître que l'on tend généralement à penser que seuls les pays développés ou les couches sociales moyennes et élevées des pays en développement sont en mesure d'adopter ces stratégies. Un des fondements de cette idée est le fait que dans les pays en développement, des processus de différenciation et des processus d'inégalité coexistent. Accepter que la personnalisation est fonction de la disponibilité des ressources fait courir le risque d'établir un fonctionnement dichotomique selon lequel les demandes des couches pauvres seraient satisfaites par des programmes de masses et celles des couches moyennes et élevées par des actions personnalisées. Personne ne peut avancer cependant, sous le prétexte de la nécessité de satisfaire aux demandes massives, que l'attention personnalisée est moins nécessaire dans les services destinés aux secteurs de revenus modestes que dans ceux desservant la population disposant d'un revenu élevé. Dans le cas de l'éducation, par exemple, il y a des preuves nombreuses et variées des attitudes différentes manifestées par les enfants provenant de familles modestes face aux exigences du processus d'apprentissage.

L'application de stratégies différenciées pour obtenir des résultats homogènes ne constitue donc pas un critère nécessaire pour atteindre des objectifs démocratiques. À cet égard, il paraît nécessaire de souligner que les orientations des changements en matière d'éducation dans les pays en développement sont étroitement liées au rôle de la coopération internationale. Comme on le sait, et sans parler de leur rareté, les ressources nationales sont généralement consacrées au paiement des salaires. La marge disponible pour investir dans la formation du personnel, les innovations pédagogiques, l'équipement, les systèmes d'information etc., est très réduite. Pour cette raison, le rôle de la coopération internationale est, du point de vue stratégique, un rôle crucial. Définir des orientations appropriées dans ce domaine constitue donc une tâche énorme de responsabilité. 


\section{NOTES}

1. Les opinions qui sont exprimées dans cet article sont la responsabilité de l'auteur et n'engagent en aucune manière ni l'UNESCO ni le BIE. L'analyse, reproduite ici avec l'accord de l'UNESCO, fait partie des études soumises à la Commission internationale sur l'éducation pour le vingt et unième siècle à sa première session (Paris, 3-5 mars 1993, (C UNESCO).

2. Source: Chubb John E., Hanushek Eric A., in Setting National Priorities: Policy for the Nineties; The Brookings Institution, Wash. D.C.

\section{RÉSUMÉS}

Ce texte tente de résumer les principales leçons que l'on peut tirer des politiques de réformes éducatives menées au cours des dernières décennies. Devant les résultats très minces, l'auteur insiste sur l'importance du consensus et du long terme comme conditions du succès.

\section{INDEX}

Mots-clés : éducation comparée, politique éducative, recherche comparative, réforme de l'enseignement, système éducatif

\section{AUTEUR}

\section{JUAN CARLOS TEDESCO}

Directeur du Bureau international d'éducation (BIE), Genève. 